\title{
Energy Saving Supply System Design Based on Wireless Sensor Network
}

\author{
Hao Sun \\ Electronic Science teaching and research room, Xi'an FanYi University Taiyigong Town, Changan \\ District, Xi'an City, Shanxi Province, China \\ 76802682@qq.com
}

\begin{abstract}
Keywords: Wireless sensor network; Energy storage sensor node; Optimal path tree; Energy consumption analysis
\end{abstract}

\begin{abstract}
Wireless sensor network (WSN) is a new generation of sensor networks, which has an extensive application prospect, but the energy limitation of sensor nodes is a key issue of WSN. This paper designs a kind of energy saving supply system which is applied to WSN. The system makes full use of the electric energy to charge up and restore the electric energy into battery. When a network node (or key node) in the system needs to be charged, the system makes use of the routing algorithm to find an optimal power supply path to charge the node in the way of wireless charging.

Firstly, the system divided sensor nodes into two types: common type and energy storage type. Secondly, according to the distribution of wireless sensor nodes in the system, the sensor nodes are divided into different areas for management and the energy management area centering on energy storage sensor nodes is established. Energy transfer path between sensor nodes among the whole network will be stored in an energy storage sensor node in the form of an optimal path tree (SPF tree) or its branch so as to achieve energy coverage for all sensor nodes. Finally, the problem of energy consumption of the system is analyzed and some solutions are put forward to extend the service life of WSN. For this system, there are still a lot of work to be done in the further optimization of energy management strategy and the further reduction of energy consumption. Further research on these problems is expected to be done in the future.
\end{abstract}

\section{Introduction}

Wireless sensor network (WSN)is a new generation of sensor networks, has a very extensive application prospect [1].Small capacity battery generally used to charge the sensor node in WSN. In most cases, the working environment is complex with wide distribution area. If the energy is used up and cannot be supplemented in time, it is very unfavorable to monitor and collect the key data on some key occasions. At present, the research on WSN energy limitation is mainly focused on the low power design of sensor nodes and the optimization of sensor node energy management strategy [2].

This paper designs a kind of energy saving supply system applied to WSN. The system makes full use of solar energy to charge the sensor nodes which has insufficient energy. If some sensor nodes (or key nodes) can not be charged with solar energy, other sensor nodes can use the routing algorithm to transmit the energy to the sensor node in a wireless way through the network.

\section{The Overall System Design}

Sensor Node Design. The sensor node of the system is composed of four modules: sensing module, processing module, RF module and power module, as shown in the following figure: 
Power module
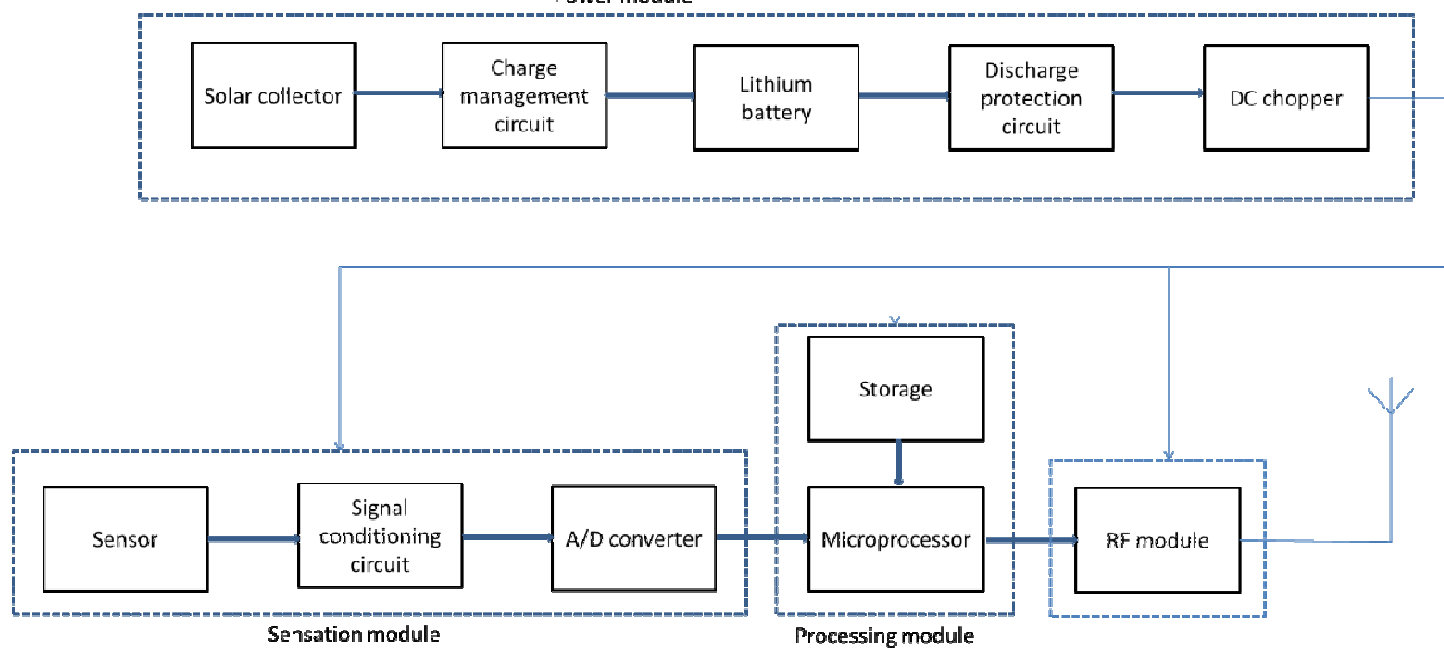

Figure 1. Structure of sensor node

In the power-supply module, the lithium battery is responsible for supplying the energy needed by the sensor node. When the energy of the sensor node falls below a certain value (such as $50 \%$ ), the sensor node opens the solar battery board to charge the lithium battery. The charging process is under the supervision of the charging management circuit so as to avoid overcharging the lithium battery. The function of the discharge protection circuit is to avoid the excessive discharge of the lithium battery. DC chopper provides a stable voltage for the load on the node on the basis of the power supply of other modules[3-4].

The sensor module includes three sub modules: sensor, signal conditioning circuit and analog / digital converter ADC. The environmental information (analog signal) collected by the sensor is processed by the signal conditioning circuit and then input to the analog / digital converter ADC. The analog / digital converter ADC converts the converted digital signals into the microprocessor for processing. The processing module processes the received data according to the requirement of the system, and sends the result to the network through the radio frequency module. Moreover, the radio frequency module transfers the received data information to the microprocessor for processing.

Region Division. How to effectively organize the sensor nodes into the network and manage the energy of the sensor nodes in the network is the most important issue for WSN [5]. In order to realize the energy transfer and supplement in the system, the sensor nodes are divided into two types: common type and energy storage type.

The structure of these two types of sensor nodes is consistent with the design of Figure 1, and the difference is that the lithium battery capacity of the sensor node of Energy storage type is significantly greater than sensor nodes of conventional type. Therefore, the energy storage sensor nodes can be taken as the center, and a certain distance can be taken as the radius, so as to wireless charge the Common sensor node within the circular region. Considering the propagation loss of electromagnetic wave in free space, the radius of the circular area theoretically covered by each energy storage sensor node should be controlled within 2 meters [6]. If a storage type sensor node needs to charge more energy than its own storage power, other energy storage type sensor nodes need to use the shortest path founded by selection algorithm to transfer energy to the target sensor node through the multi hop [7-8]. So in the process of designing routing algorithm, each "Hop" in the process of energy transfer, should first selects the nearest storage type sensor node. Only when it is far away from the adjacent energy storage type sensor node (such as more than 2 meters), the common sensor node can be considered as next hop of energy transfer.

Therefore, in the formulation of the routing protocol, bit binary number should be used in identifier of the sensor node to distinguish the type of the node, which means that the " 0 " can be used to represent the common sensor nodes, and the "1" represents the energy storage type sensor nodes. When these sensor nodes began self-organizing into wireless Sensor Networks after being arranged, each sensor node will package its own parameters (including identifier, location, etc.) into 
a packet to send to the broadcast channel. Routing selection algorithm such as the shortest pathfirst algorithmcan (SPF) be used here. A specified energy storage type sensor node can be taken as root node (the root node should be located in the center position of the coverage area of the wireless sensor network as far as possible). A shortest path from the root node to each energy storage sensor node should be calculated to generate the shortest path tree. When the energy transfer is needed, the energy transfer is carried along the route of the shortest path tree. When the adjacent energy storage sensor nodes are far away from each other, the common sensor nodes can be used as the node in the SPF tree.

After SPF tree is generated, the common sensor nodes can establish the adjacency relationship with the nearest energy storage type sensor node. Under normal circumstances, they are charged by solar panels for power supplement. Under some special circumstances (such as solar panels cannot work), they are charged by the energy storage sensor node for power supplement. The common sensor nodes, which are far away from any energy storage type sensor nodes, must inform the nearest 2-3 sensors other common sensor nodes through network. Under the condition that this kind of sensor nodes must be charged, the energy storage type sensor nodes can use the common sensor nodes which are notified to the network as relay node to supplement this sensor node with energy. The energy transfer path generated under the above conditions can be taken as a supplementary branch of the SPF tree and recorded in each storage type sensor node.

Using the regionalization method of energy management described above, this system will do its best to cover all sensor nodes with energy in the whole wireless sensor network, so as to realize the energy supplement to any key nodes in the critical condition.

\section{Analysis and Countermeasures of System Energy Consumption}

In addition to the power module generating energy in this system, the other three modules are the energy consumption source of the sensor node. The simple analysis on the energy consumption composition of these three modules is as follows.

Sensor Module. The energy consumption of the sensor module is mainly related to the following three factors: The type of measurement of physical quantities (temperature, sound or image), the length of the induction time and the complexity of the surrounding environment. The energy consumed by the sensor module is much less than the energy consumed by the RF module. The low power consumption of the hardware circuit of the sensor node can be designed to further reduce the energy consumption of the sensor module.

Processing Module. When processing module is processing data, the energy consumption is much less than the energy consumption of RF module [9]. In this system, we can use the data fusion technology (especially the data gathered in the energy storage sensor node) for the data on the sensor nodes. The transmission of data can be greatly reduced by increasing a little energy consumption in processor, thus further reducing energy consumption. Beamforming algorithm is a typical data fusion algorithm [10].At the same time, when the calculated load of processing module is low, the dynamic voltage regulation (DVS) technology can be used[11].

Radio Frequency Module. There are four kinds of working states of RF module: send, receive, idle and sleep. When the radio frequency module is in the idle state, the communication status of wireless channel is always monitored to check whether the data is sent to the user himself. Turn off radio frequency module when it is in sleep state, so the energy consumption is very low. The research shows that when sending and receiving data, especially when transmitting data, the radio frequency module has the most energy consumption[12]. Moreover, the radio frequency module cannot be idle for a long time, because it will increase the energy consumption of sensor nodes [13].

Therefore, if we want to further reduce the energy consumption of the system, we should use dynamic energy management (DPM) technology. According to the requirements of the SPF tree and the system generated in the region, the working state of each energy storage sensor node is determined dynamically. The working state of the common sensor node is consistent with the nearest energy storage sensor node (or other common sensor nodes in the SPF tree). When there is work requirement, the sensor node is in a operative state (or the sleep state is waken up to become 
the operative state); When there is no work requirement, the sensor node should be in the state of sleep. At the same time, it is necessary to reduce the idle time of the sensor node in order to minimize the energy consumption of the RF module.

\section{Conclusion}

At present, wireless sensor network (WSN) is more and more widely used in the production and life of the society. The limitation of the energy of sensor nodes has always been the hot topic of the research. This paper designs a kind of energy saving supply system applied to WSN. In this system, the sensor nodes are specifically divided into two types: common type and energy storage type. In this system, the energy management area which centers on energy storage sensor node is established. Energy transfer path between each sensor node in the whole network is stored in the storage type sensor node in the form of an optimal path tree (SPF tree) or its branch, so as to achieve coverage of the energy of all sensor nodes. The system use a low power consumption design and energy management strategy to further reduce the energy consumption of the whole system, and finally extend the service life of the WSN. There are still a lot of work to be done in the further optimization of energy management strategy and the further reduction of energy consumption. Further research on these problems is expected to be done in the future.

\section{Acknowledgements}

Thesis project: 2015 research project of Xi'an FanYi University Project number: 15B04

\section{References}

[1] Wireless sensor network [M]. Electronic Industry Press, (American)lan F. Akyildiz Mehmet Can Vuran series, Xu Pingping, Liu Hao, translated by, 2013, 3.(In Chinese)

[2] Research on wireless sensor network technology [J]. Chen Ying, Shu Jian, Chen Yubin, Yin min. Sensors and microsystems. 2007 (10).(In Chinese)

[3] Solar photovoltaic technology [M]. Xi'an Jiao Tong University press, (Germany) Wagemann (Wagemann), 2011.(In Chinese)

[4] Study on solar photovoltaic power generation system application [J]. Dong You'er, Meng Yu, Shen Tiantian, Tang Jin'e. Journal of Shanxi University (NATURAL SCIENCE EDITION). 2013 (01). (In Chinese)

[5]A1: An energy efficient topology control algorithm for connected area coverage in wireless sensor networks[J]. Sajjad Rizvi, Hassaan Khaliq Qureshi, Syed Ali Khayam, Veselin Rakocevic, Muttukrishnan Rajarajan. Journal of Network and Computer Applications . 2011 (2).

[6] Review of energy optimization of wireless sensor networks [J]. Lu Guiyan, Wang Lingfen, Mi Shoufang. Journal of Dalian Nationalities University. 2013 (03).(In Chinese)

[7] Energy Efficient Multicast Routing Protocols for Wireless Sensor Networks. A.A. Minihas, D. Satter,K. Mustaq, S.A. Rizvi. 2011 World Congress on Sustainable Technologies . 2011

[8] Comparative analysis of classical routing protocol leach and its updated variants that improved network life time by addressing shortcomings in wireless sensor network. HANEEF M,DENG ZH L. Seventh International Conference on Mobile Ad-Hoc and Sensor Networks . 2011

[9] Hamrita T K, Kaluskar N P and Wolfe K.L. Advances in smart sensor technology[C].Conference of Industry Applications,2005,3:2059-2062.

[10] MUDUM BAT R,BARRTAC G,MADHOW U.On the feasibility of distrbuted beamforming in wireless networks[J].IEEE Trans Wireless Commun, 2007, 6(5):1754-1763.

[11] Reason J M and Rabaey J M.A study of energy consumption and reliability in a multi-hop sensor networks[J].ACM SIGMOBILE Mobile Computing and Communications Review,2004,8(1):84-97.

[12] Lm C,Kim H,Ha S. Dynamic Voltage Scheduling Technique for Low-power Multimedia 
Application Using Buffers// Proceedings of the International Symposium on Low Power Electronies and Design. California: ACM Portal Press,2001:34-39.

[13] Miller M J and Vaidya N H. Minimizing energy consumption in sensor networks using a wakeup radio[C].Proceeding of the IEEE Wireless Communications and Networking, WCNC'04, Atlanta,GA,2004,4:2335-2340. 